\section{DISCUSSION}

The technique of clamping the PA and PV of the reserved lobe for PA reconstruction can provide clearer surgical vision, greater operative space, simpler procedures, and safer margins, as previously described elsewhere. ${ }^{1}$ We transferred this technique into VATS, where it has made PA reconstruction both more feasible and more easily performed.

When performing PA reconstruction in VATS, as described in previous reports, ${ }^{2,3}$ the vascular clamp has been inserted through the $40-\mathrm{mm}$ access incision. When this is done, the clamp obviously occupies the already limited space and increases the difficulty for surgeons. To gain more space, we added a $12-\mathrm{mm}$ incision specifically for the PA clamping. More importantly, by using an umbilical tape and a 5-mm clip, another incision for PV occlusion was avoided as well. The PA reconstruction thus could be performed much more safely in a larger space.

Although the limitations of this study include the small number of patients and the short-term nature of our results, our most current data show our technique to be feasible and safe when applied in VATS lobectomy with partial removal of the PA. Relative to a conventional PA reconstruction strategy in VATS, ${ }^{2,4,5}$ our technique has several advantages. (1) An additional incision for PA clamping can provide more space. (2) An additional incision for PV occlusion can be avoided. (3) Blocking the PV of the reserved lobe with a tape instead of a vascular clamp can provide clearer surgical vision, greater operative space, and safer margins.
An important limitation of this study concerns the extent of reconstruction. It is notable that the 5 patients in our series underwent only partial PA reconstruction with direct suturing, and the infiltration usually involved less than $25 \%$ of the circumference of the PA. In cases where more than $40 \%$ of the circumference is invaded, a patch repair or sleeve resection would be inevitable. In the future, we will be attempting such complex surgical procedures in VATS with the aid of our new technique.

In conclusion, this technique can reduce the risk of intraoperative bleeding and the chance of needing a conversion to open thoracotomy. Application of this technique thus may allow us to extend the inclusion criteria for VATS lobectomy.

\section{References}

1. Yin R, Xu L, Ren B, Jiang F, Fan X, Zhang Z, et al. Clinical experience of lobectomy with pulmonary artery reconstruction for central non-small-cell lung cancer. Clin Lung Cancer. 2010;11:120-5.

2. Nakanishi R, Yamashita T, Oka S. Initial experience of video-assisted thoracic surgery lobectomy with partial removal of the pulmonary artery. Interact Cardiovasc Thorac Surg. 2008;7:996-1000.

3. Kamiyoshihara M, Nagashima T, Ibe T, Takeyoshi I. A tip for controlling the main pulmonary artery during video-assisted thoracic major pulmonary resection: the outside-field vascular clamping technique. Interact Cardiovasc Thorac Surg. 2010;11:693-5.

4. Nakanishi R, Oka S, Odate S. Video-assisted thoracic surgery major pulmonary resection requiring control of the main pulmonary artery. Interact Cardiovasc Thorac Surg. 2009;9:618-22.

5. Amer K, Khan AZ, Vohra HA. Video-assisted thoracic surgery of major pulmonary resections for lung cancer: the Southampton experience. Eur J Cardiothorac Surg. 2011;39:173-9.

\title{
Video-assisted thoracoscopic surgery with spontaneous breathing laryngeal mask anesthesia: Preliminary experience
}

\author{
Marcello Carlo Ambrogi, MD, PhD, ${ }^{\text {a }}$ Olivia Fanucchi, MD, ${ }^{\text {a }}$ Raffaello Gemignani, MD, ${ }^{\text {b }}$ \\ Fabio Guarracino, MD, ${ }^{\mathrm{b}}$ and Alfredo Mussi, MD, ${ }^{\mathrm{a}}$ Pisa, Italy
}

Video clip is available online.

\footnotetext{
From the Divisions of Thoracic Surgery ${ }^{\mathrm{a}}$ and Cardiothoracic Anaesthesia and Intensive Care, ${ }^{\mathrm{b}}$ CardioThoracic and Vascular Department, University of Pisa, Pisa, Italy.

Disclosures: Authors have nothing to disclose with regard to commercial support. Received for publication Sept 22, 2011; revisions received Jan 16, 2012; accepted for publication Jan 25, 2012; available ahead of print Feb 23, 2012.

Address for reprints: Olivia Fanucchi, MD, Cardiac Thoracic and Vascular Department, via Paradisa 2, 56124, Pisa, Italy (E-mail: olivia.fanucchi@for.unipi.it).

J Thorac Cardiovasc Surg 2012;144:514-5

$0022-5223 / \$ 36.00$

Copyright (c) 2012 by The American Association for Thoracic Surgery

doi:10.1016/j.jtcvs.2012.01.077
}

General anesthesia with single-lung ventilation is generally considered mandatory for VATS procedures. Recently, in an attempt to avoid tracheal intubation-related risks, ${ }^{1,2}$ some authors have reported their experience with awake thoracic surgery under sole thoracic epidural anesthesia. $^{3,4}$ They have shown that several minor thoracoscopic operations can be performed under spontaneous breathing, without general anesthesia with single-lung ventilation. Nevertheless, this procedure also presents some adverse events: pain, panic attack, cough reflex during lung parenchyma manipulation, and potentially serious complications related to epidural anesthesia. We reasoned that to avoid both single-lung ventilation and epidural anesthesia risks, thoracoscopic lung resections could 
be performed with patients under general anesthesia and spontaneously breathing with laryngeal mask anesthesia (LMA).

\section{MATERIALS AND METHODS}

The main intent of this study was to evaluate the safety and technical feasibility of VATS pulmonary resection with LMA. For this experience, we initially chose the relatively simple procedure of apicetomy for the treatment of primary spontaneous pneumothorax, which traditionally carries only a low conversion to open surgery rate. The study was approved by our institutional review board. All patients signed a detailed informed consent form.

The only contraindication for LMA was the presence of congenital or acquired oropharyngeal malformations.

LMA was carried out under total intravenous technique with propofol and fentanyl. No muscle relaxants were used throughout the procedure, thereby permitting spontaneous breathing of a $50 \%$ oxygen-air mixture (Video 1). The patients' parameters were continuously and noninvasively monitored.

All procedures were performed with the patient in the lateral decubitus position. The level of technical feasibility was defined by the operating surgeon and stratified into 4 grades: unsatisfactory, satisfactory, good, or excellent, according to the surgeon's personal experience with traditional anesthesia. The maximum and minimum values of end-tidal $\mathrm{PCO}_{2}$ and arterial oxygen saturation were recorded during the procedure.

\section{RESULTS}

Eight consecutive patients (6 male, 2 female) with a mean age of 31.3 years (range, 26-39 years) underwent apicectomy by mechanical stapler followed by pleural abrasion. All had a Vanderschueren stage III or IV pneumothorax without significant associated comorbidities. In all cases, a complete collapse of nondependent lung was obtained, and lung manipulation and parenchymal resection were easily performed (Video 2 ). Technical feasibility was considered excellent in all cases. No intraoperative complications or laryngeal mask displacements occurred. One patient required several minutes of assisted ventilation by laryngeal mask for drug-related breathing depression. The values of arterial oxygen saturation, and minimum and maximum end-tidal carbon dioxide tension resulted excellent during the entire procedure for all patients: they were $99.6 \%$ (range, 99\%-100\%), 34.6 $\mathrm{mm} \mathrm{Hg}$ (range, 32-37 mm Hg), and $38.6 \mathrm{~mm} \mathrm{Hg}$ (range, 36-40 mm Hg), respectively. No postoperative complications occurred. Mean drainage period and hospital stay were 2.4 days (range, 2-3 days) and 3.3 days (range, 3-4 days), respectively. At a mean follow-up of 17.7 months (range, 10-25 months), there were no pneumothorax recurrences.

\section{DISCUSSION}

Double-lumen intubation with general anesthesia has permitted undoubted and well-known improvement, although it is associated with potential complications related to tracheal intubation and mechanical ventilation. ${ }^{1,2}$ Our results suggest that pulmonary parenchymal resection can be feasibly and safely performed with general anesthesia even in spontaneous breathing through the use of LMA. The nondependent lung in fact undergoes a complete deflation when an open pneumothorax is created after the first thoracoscopic access. An adequate operating space is therefore available for movement of thoracoscopic instruments. Additionally, the excellent level of feasibility defined by the surgeon in every case demonstrated that the parenchyma can be manipulated easily without any cough reflex that could hamper its surgical resection. This was described by Pompeo and colleagues ${ }^{3,4}$ with awake epidural technique in $14 \%$ of cases. Their patients also had trocar site pain in $9.5 \%$ of cases and panic attack in $6 \%$ to $9.5 \%$ of cases, both of which were completely absent in our experience with LMA. Moreover, LMA is free from serious potential risks related to epidural hematoma or spinal cord injury. More recently, Katlic and associates ${ }^{5}$ reported excellent results in a large series with deep sedation and local anesthesia without tracheal intubation or epidural anesthesia; however, their high dosage of fentanyl seems to carry the potential for breathing depression, as happened in 1 patient in our series. In our case, this complication was easily managed thanks to the laryngeal mask.

The main limitations of our study are the subjective evaluation of technical feasibility and above all the limited series. Despite these, this first experience seems to be favorable for LMA in VATS procedures, avoiding the risks associated with tracheal intubation and mechanical ventilation, permitting safe anesthesia without pain or panic attack, and allowing confident manipulation of the pulmonary parenchyma without cough. On the basis of these preliminary results, we surmise that LMA can be also applied to older patients with chronic obstructive pulmonary disease and other comorbidities, for whom its advantages would be even more considerable.

\section{References}

1. Miñambres E, Burón J, Ballesteros MA, Llorca J, Muñoz P, González-Castro A. Tracheal rupture after endotracheal intubation: a literature systematic review. Eur J Cardiothorac Surg. 2009;35:1056-62.

2. Tokis L, Hedensttierna G, Svensson L, Brismar B, Cederlund T, Lundquist H, et al. V/Q distribution and correlation to atelectasis in the anesthetize paralyzed humans. J Appl Physiol. 1996;81:1822-33.

3. Pompeo E, Mineo D, Rogliani P, Sabato AF, Mineo TC. Feasibility and results of awake thoracoscopic resection of solitary pulmonary nodules. Ann Thorac Surg. 2004;78:1761-8.

4. Pompeo E, Mineo TC. Awake pulmonary metastasectomy. J Thorac Cardiovasc Surg. 2007;133:960-6.

5. Katlic MR, Facktor MA. Video-assisted thoracic surgery utilizing local anesthesia and sedation: 384 consecutive cases. Ann Thorac Surg. 2010;90:240-5. 